\title{
HARMONIC ANALYSIS ON GRASSMANNIAN BUNDLES
}

\author{
ROBERT S. STRICHARTZ ${ }^{1}$
}

\begin{abstract}
The harmonic analysis of the Grassmannian bundle of $k$-dimensional affine subspaces of $\mathbf{R}^{n}$, as a homogeneous space of the Euclidean motion group, is given explicitly. This is used to obtain the diagonalization of various generalizations of the Radon transform between such bundles. In abstract form, the same technique gives the Plancherel formula for any unitary representation of a semidirect product $G \times V$ ( $V$ a normal abelian subgroup) induced from an irreducible unitary representation of a subgroup of the form $H \times W$.
\end{abstract}

Introduction. Let $G_{n, k}$ denote the Grassmannian manifold of linear $k$-dimensional subspaces of $\mathbf{R}^{n}$, and let $P_{n, k}$ denote the Grassmannian bundle of affine $k$ dimensional subspaces (also called $k$-planes), so $P_{n, k}$ is a bundle over $G_{n, k}$ with fibre dimension $m$, where $m+k=n$. In group-theoretic terms, $G_{n, k}=G / H$, where $G=S O(n)$ and $H=S(O(k) \times O(m))$, or we could just as well take $G=O(n)$ and $H=O(k) \times O(m)$. Let $\rho$ denote the standard representation of $G$ on $V=\mathbf{R}^{n}$ and form the semidirect product $G \times V$, the Euclidean motion group, with group law $\left(g_{1}, x_{1}\right) \circ(g, x)=\left(g_{1} g, x_{1}+\rho\left(g_{1}\right) x\right)$. The Eucidean motion group acts naturally on the bundle $P_{n, k}$. If we let $\pi_{0}$ denote the subspace spanned by the first $k$ basis elements $e_{1}, \ldots, e_{k}$, then the isotropy subgroup of $\pi_{0}$ is $H \times W$ where $W$ is the subspace $\pi_{0}$. Thus $P_{n, k}=G \ltimes V / H \times W$.

In this paper we obtain the explicit harmonic analysis on $P_{n, k}$, the decomposition of $L^{2}\left(P_{n, k}\right)$ (with respect to the invariant measure) into a direct integral of irreducible representations of $G \times V$. This is accomplished in two stages. In the first abstract stage ( $\$ 2)$ we let $G$ be any compact Lie group and $\rho$ any real representation of $G$ on $V$, and for functions $f(g, x)$ invariant under the right action of a subgroup $H \times W$ we obtain the Fourier decomposition formula

$$
f(g, x)=\int_{\Lambda} \sum_{\delta \in \Delta_{\lambda}} F_{\lambda, \delta}(g, x) d \nu(\lambda) .
$$

Here $\Lambda$ is an index set for the orbits in $W^{\perp}$ under the action of $H$. If we choose a point $z_{\lambda}$ in each orbit and let $G_{\lambda}$ and $H_{\lambda}$ denote the isotropy subgroup of $z_{\lambda}$ in $G$ and $H$ respectively, then $\Delta_{\lambda}$ denotes the set of irreducible representations of $G_{\lambda}$ that occur in $L^{2}\left(G_{\lambda} / H_{\lambda}\right)$. The measure $d \nu(\lambda)$ is determined by $d z=d \mu_{\lambda} d \nu(\lambda)$, where $d z$ is Lebesgue measure on $W^{\perp}$ and $d \mu_{\lambda}$ is the invariant measure on the orbit.

Received by the editors March 7, 1985.

1980 Mathematics Subject Classification. Primary 43A85; Secondary 22D30, 44A15.

Key words and phrases. Grassmannian bundle, induced representation, semidirect product, harmonic analysis, generalized Radon transform, Mackey theory, affine symmetric space, Euclidean motion group, integral geometry.

${ }^{1}$ Research supported in part by National Science Foundation Grant DMS-8401354. 
The space of Fourier components $F_{\lambda, \delta}$ for fixed $\lambda, \delta$ (for $\delta \in \Delta_{\lambda}$ ) realizes a primary (or irreducible) representation of $G \times V$ induced from a primary (or irreducible) representation of $G_{\lambda} \times V$ associated to the parameters $\delta$ and $z_{\lambda}$ by the Mackey theory. The expression for $F_{\lambda, \delta}$ in terms of $f$ is

$$
\begin{aligned}
F_{\lambda, g}(g, x)=(2 \pi)^{-m} \int_{H} \int_{G_{\lambda}} \int_{W^{\perp}} f\left(g h g_{\lambda}^{-1}, \rho\left(g h g_{\lambda}^{-1}\right)(0, z)\right) \\
\times K_{\lambda, \delta}\left(g_{\lambda}\right) e^{-i\left\langle z, z_{\lambda}\right\rangle} e^{i\left\langle x, \rho(g h) z_{\lambda}\right\rangle} d z d g_{\lambda} d h,
\end{aligned}
$$

where $K_{\lambda, \delta}\left(g_{\lambda}\right)$ is the bi- $H_{\lambda}$-invariant zonal function on $G_{\lambda}$ associated to the representation $\delta$.

In the second explicit stage ( $(3)$, we return to the case of $P_{n, k}$ and seek an explicit formula for one typical Fourier component $F_{\lambda, \delta}$ for each $\lambda, \delta$. What we obtain is (for a particular choice of $f$ )

$$
F_{\lambda, \delta}(g, x)=\int_{O(m)} M_{\delta}\left(g\left(\begin{array}{l}
0 \\
s
\end{array}\right)\right) e^{i \lambda\left\langle x, g\left(s e_{n}\right)\right\rangle} d s,
$$

where $M_{\delta}\left(\omega_{1}, \ldots, \omega_{m}\right)$ is an explicit function defined in terms of certain determinants and $\left(s e_{n}\right)$ means we let $s \in O(m)$ act on the last $m$ components. This is analogous to our previous description of harmonic analysis on $G_{n, k}$ in [10]. The function $F_{\lambda, \delta}$ obtained is "marked" in that it lies in a certain one-dimensional subspace, a highest weight subspace for a certain irreducible representation of $G$ which occurs with multiplicity one in the representation induced from $\delta$ on $G_{\lambda}$. Thus any intertwining operator from functions on $P_{n, k^{\prime}}$ to functions on $P_{n, k}$ must send each such $F_{\lambda, \delta}^{\prime}$ on $P_{n, k^{\prime}}$ to a multiple of $F_{\lambda, \delta}$ on $P_{n, k}$.

This is extremely useful in applications. As an illustration ( $\$ 5)$ we diagonalize certain generalizations of the Radon transform. We fix $k^{\prime}$ and $k$, and we choose an integer parameter $j$ with $\max \left(0, k+k^{\prime}-n\right) \leq j \leq \min \left(k, k^{\prime}\right)$. Then define $R\left(k^{\prime}, k, j\right)$ from functions on $P_{n, k^{\prime}}$ to functions on $P_{n, k}$ by $R\left(k^{\prime}, k, j\right) f(\pi)=\int f\left(\pi^{\prime}\right)$, where the integral is taken over all $k^{\prime}$-planes $\pi^{\prime}$ that intersect the fixed $k$-plane $\pi$ orthogonally in a $j$-dimensional set. These transformations include the usual $k$-plane transform and its dual, and also certain transforms considered by Gonzalez [3]. For those values of $\delta$ which occur in both $P_{n, k^{\prime}}$ and $P_{n, k}$, we have

$$
R\left(k^{\prime}, k, j\right) F_{\lambda, \delta}^{\prime}=\lambda^{j-k} c(\delta) F_{\lambda, \delta},
$$

where $c(\delta)$ is given as the ratio of certain compact integrals. Of course $R\left(k^{\prime}, k, j\right) F_{\lambda, \delta}^{\prime}$ $=0$ if $\delta$ occurs in $P_{n, k^{\prime}}$ but not $P_{n, k}$, and this happens when $\min \left(k^{\prime}, n-k^{\prime}-1\right)$ $>\min (k, n-k-1)$. It seems likely that this approach will yield an inversion formula for the remaining cases if it is possible to compute the factors $c(\delta)$ more explicitly.

The abstract harmonic analysis in $\S 2$ is extended in $\S 4$ to include more general induced representations of semidirect products. Let $G \times V$ denote a semidirect product, where $V$ is a locally compact abelian group, $G$ is a locally compact group, and $\rho$ is a continuous homomorphism of $G$ into the automorphisms of $V$, with the group law as before. Consider any closed subgroup of the form $H \times W$ and any representation of $G \times V$ induced from an irreducible representation of $H \times W$. We assume some weak measure-theoretic regularity, so the Mackey theory applies. By passing to a smaller subgroup if necessary, we may assume without loss of generality that the representation we are inducing from has the form $\pi \times \chi$, where $\pi$ is an 
irreducible unitary representation of $H$ on a Hilbert space $\not$ and $\chi$ is a character of $W$ such that $\rho(H) \chi=\chi$.

This time there are three parameters involved in the harmonic analysis of a function $f \in L^{2}(G \times V, H, \pi \times \chi)$, the representation space of the induced representation. First set $Z=V / W$, decompose $\hat{Z}$ under the action $\rho(H)$ into orbits, and let $\Lambda$ be a parameter space for the orbits, with $\zeta_{\lambda}$ a representative point in each orbit. As before let $G_{\lambda}$ and $H_{\lambda}$ denote the isotropy subgroup of $\zeta_{\lambda}$ in $G$ and $H$, respectively. Second, for each fixed $\lambda$, restrict $\pi$ to $H_{\lambda}$ and decompose into a direct integral $\int_{A} \pi_{\alpha} d \alpha$ of primary (or irreducible) representations $\left(\pi_{\alpha}\right.$ denotes such a representation of $H_{\lambda}$ on $H_{\alpha}$ ). Third, for each fixed $\lambda$ and $\alpha$, decompose the representation of $G_{\lambda}$ induced from the representation $\pi_{\alpha}$ of $H_{\lambda}$ into a direct integral $\int_{\Delta_{\lambda, \alpha}} \pi_{\lambda, \alpha, \delta} d \delta$ of primary (or irreducible) representations of $G_{\lambda}$. Then the Fourier decomposition is

$$
f=\int_{\Lambda} \int_{A} \int_{\Delta_{\lambda, \alpha}} F_{\lambda, \alpha, \delta} d \delta d \alpha d \nu(\lambda),
$$

where the Fourier component $F_{\lambda, \alpha, \delta}$ lies in a space of functions equivalent to the representation space for $G \times V$ of the representation induced from $\pi_{\lambda, \alpha, \delta} \times\left(\chi+\varsigma_{\lambda}\right)$ on $G_{\lambda} \times V$.

The paper concludes $(\S 6)$ with some remarks, including some open problems that appear interesting.

The motivation for studying harmonic analysis on Grassmannian bundles, aside from its intrinsic interest and applications to Radon transforms, comes from the theory of affine symmetric spaces. Recall that an affine symmetric space is defined to be a homogeneous space of a Lie group where the factor group is the fixed point set (or a union of connected components of it) of an involution (see Berger [1]). Now the harmonic analysis of compact symmetric spaces, such as $G_{n, k}$, is well known, as is the case of noncompact Riemannian symmetric space $(G / K, G$ semisimple, $K$ maximal compact). Recently, considerable effort has gone into trying to understand semisimple symmetric spaces, where $G$ is assumed semisimple. See Flensted-Jensen [2] and Oshima [9] for expositions of the current state of knowledge, which is very incomplete. The semisimple symmetric spaces possess the additional structure of an invariant semi-Riemannian metric (it is the geodesic symmetries with repect to this metric that explain the nomenclature "symmetric").

Now the Grassmannian bundles are examples of affine symmetric spaces of a degenerate sort; in particular, they possess no invariant semi-Riemannian metrics (this is discussed in $\S 6$ ). But they can be realized as limits of compact or semisimple symmetric spaces. To explain the compact limit we may regard $G_{n+1, k+1}$ as the space of $k$-planes in the $n$-sphere $S^{n}$ (here $k$-plane means totally geodesic $k$-dimensional submanifold) by embedding $S^{n}$ in $\mathbf{R}^{n+1}$ in the usual way and intersecting the $(k+1)$-planes in $\mathbf{R}^{n+1}$ with $S^{n}$. Then by realizing $\mathbf{R}^{n}$ as the limit of spheres of radii $\rightarrow \infty$ we obtain $P_{n, k}$ as a limit of $G_{n+1, k+1}$. But we can also realize $\mathbf{R}^{n}$ as the limit of hyperbolic $n$-spaces with curvature $\rightarrow 0$ and so obtain $P_{n, k}$ as a limit of $O(n, 1) / O(k, 1) \times O(m)$. Thus the results of this paper may give some indication of the kind of phenomena that might arise in the harmonic analysis of these hyperbolic semisimple symmetric spaces.

The author is grateful to S. Helgason for many useful suggestions. 
2. An abstract Plancherel formula. Let $G$ be a compact Lie group, $V$ a finite-dimensional real vector space, and $\rho$ a representation of $G$ on $V$. On the Cartesian product $G \times V$ define a semidirect group product by

$$
(g, x) \circ\left(g_{1}, x_{1}\right)=\left(g g_{1}, x+\rho(g) x_{1}\right) .
$$

Let $H$ be a closed subgroup of $G$ and $W$ a subspace of $V$ such that $\rho(H) W \subseteq W$. Then $H \times W$ is a subgroup of $G \times V$. The goal of this section is to obtain the Fourier decomposition of $L^{2}(G \times V / H \times W)$ with respect to the invariant measure into a direct integral of primary components.

We may equip $V$ with an inner product $\langle x, y\rangle$ which makes $\pi$ an orthogonal representation, and then we may identify $V$ with its dual. Write $V=W \oplus W^{\perp}$ and $x=(y, z)$ for $x \in V, y \in W, z \in W^{\perp}$. A function $f$ on $G \times V / H \times W$ can be identified with a function on $G \times V$ satisfying $f(g, x)=f(g h, z+\rho(g)(y, 0))$ for all $h \in H, y \in W$. It is uniquely determined by its values at points $(g, \rho(g)(0, z))$ for $z \in W^{\perp}$, and we put

$$
\|f\|_{2}^{2}=\int_{G} \int_{W^{\perp}} \mid f(g, \rho(g)(0, z))^{2} d z d g .
$$

The harmonic analysis on $G \times V / H \times W$ proceeds in two stages; in the first stage we take the Euclidean Fourier transform on $W^{\perp}$ and group terms in $\rho(H)$ orbits. Thus write $W^{\perp}=\bigcup_{\lambda \in \Lambda} Z_{\lambda}$, where each $Z_{\lambda}$ is a $\rho(H)$ orbit in $W^{\perp}$ and $\Lambda$ is any convenient parameter set. Each orbit $Z_{\lambda}$ supports a measure $d \mu_{\lambda}$ which is invariant under $\rho(H)$, again normalized to have total mass one. Then there exists a unique measure $d \nu(\lambda)$ on $\Lambda$ such that $d z=d \mu_{\lambda} d \nu(\lambda)$.

Now take $f$ in $L^{2}(G \times V / H \times W)$, and assume first that $f$ is $C^{\infty}$ with compact support. Fix $g$ and define

$$
\hat{f}(g, \varsigma)=(2 \pi)^{-m} \int_{W^{\perp}} f(g, \rho(g)(0, z)) e^{-i\langle z, \varsigma\rangle} d z,
$$

where $\varsigma \in W^{\perp}$ and $m=\operatorname{dim} W^{\perp}$. Then

$$
f(g, \rho(g)(0, z))=\int_{W^{\perp}} \hat{f}(g, \zeta) e^{i\langle z, \zeta\rangle} d \zeta
$$

by Euclidean Fourier inversion, hence

$$
\begin{aligned}
f(g, x) & =\int_{W^{\perp}} \hat{f}(g, \zeta) e^{i\langle x, \rho(g)(0, \zeta)\rangle} d \zeta \\
& =\int_{\Lambda} F_{\lambda}(g, x) d \nu(\lambda),
\end{aligned}
$$

where

$$
F_{\lambda}(g, x)=\int_{Z_{\lambda}} \hat{f}(g, \zeta) e^{i\langle x, \rho(g)(0, \varsigma)\rangle} d \mu_{\lambda}(\varsigma) .
$$

Note that under the left action of $\left(g_{1}, x_{1}\right)$ in $G \times V, F_{\lambda}$ is sent to

$$
\int_{Z_{\lambda}}\left(\hat{f}\left(g_{1} g, \zeta\right) e^{i\left\langle x_{1}, \rho\left(g_{1} g\right)(0, \varsigma)\right\rangle}\right) e^{i\langle z, \zeta\rangle} d \mu_{\lambda}(\varsigma)
$$


for $x=\rho(g)(0, \varsigma)$, so the decomposition commutes with the group action. Also note that $\hat{f}(g, \zeta)=\hat{f}\left(g h^{-1}, \rho(h) \varsigma\right)$ for all $h \in H$ and

$$
\|f\|_{2}^{2}=(2 \pi)^{m} \int_{G} \int_{W^{\perp}}|\hat{f}(g, \zeta)|^{2} d g d \varsigma
$$

by the Plancherel theorem. For a general function $f$ in $L^{2}(G \times V / H \times W)$ we approximate by functions in $C_{\mathrm{com}}^{\infty}$ and pass to the limit; $(2.2)-(2.5)$ continue to hold in the appropriate sense. Furthermore the map $f \rightarrow \hat{f}$ is onto all $L^{2}$ functions satisfying $\hat{f}\left(g h^{-1}, \rho(h) \zeta\right)=\hat{f}(g, \zeta)$ for all $h \in H$.

In the second stage we need to decompose the functions $F_{\lambda}$ further. For each orbit $Z_{\lambda}$ choose a point $z_{\lambda} \in Z_{\lambda}$, and let $H_{\lambda}$ and $G_{\lambda}$ denote the subgroups of $H$ and $G$ that fix $z_{\lambda}$. Then the $\rho(H)$-orbit $Z_{\lambda}$ can be identified with $H / H_{\lambda}$ and $Z_{\lambda} \subseteq X_{\lambda}$, where $X_{\lambda}$ is a $\rho(G)$-orbit in $W$ that can be identified with $G / G_{\lambda}$. There is usually a "nice" way to choose $z_{\lambda}$ as $\lambda$ varies so as to minimize the number of different $H_{\lambda}$ and $G_{\lambda}$ groups.

We then define $\phi_{\lambda}\left(g H_{\lambda}\right)=\hat{f}\left(g, z_{\lambda}\right)$, so $\phi_{\lambda}$ is a function on $G / H_{\lambda}$ and $\phi_{\lambda}$ determines $\hat{f}(g, \zeta)$ for all $\zeta \in Z_{\lambda}$. In terms of $\phi_{\lambda}$ we have

$$
F_{\lambda}(g, x)=\int_{H} \phi_{\lambda}(g h) e^{i\left\langle x, \rho(g h) z_{\lambda}\right\rangle} d h,
$$

and the left action of $\left(g_{1}, x_{1}\right)$ on $F_{\lambda}$ intertwines the action

$$
\phi_{\lambda} \rightarrow \phi_{\lambda}\left(g_{1} g\right) e^{i\left\langle x_{1}, \rho\left(g_{1} g\right) z_{\lambda}\right\rangle} \text {. }
$$

Furthermore, by (2.5) we have $\|f\|_{2}^{2}=(2 \pi)^{m} \int_{\Lambda}\left\|\phi_{\lambda}\right\|_{2}^{2} d \nu(\lambda)$, where $\left\|\phi_{\lambda}\right\|_{2}$ denotes the $L^{2}$ norm on $G / H_{\lambda}$, and the mapping $f \rightarrow \phi_{\lambda}$ is onto $L^{2}$ sections $\lambda \rightarrow L^{2}\left(G / H_{\lambda}\right)$.

If we were to ignore the action of $V$, we would be asking for the Fourier decomposition of $L^{2}\left(G / H_{\lambda}\right)$, or equivalently the representation induced from the trivial representation of $H_{\lambda}$. The action of $V$ requires that we induce in stages from $H_{\lambda}$ to $G_{\lambda}$ and then from $G_{\lambda}$ to $G$. Of course the representations induced from irreducible representations of $G_{\lambda}$ extend to irreducible representations of $G \times V$ according to Mackey's theory [8].

Thus let $\delta$ denote any irreducible representation of $G_{\lambda}$ that occurs in $L^{2}\left(G_{\lambda} / H_{\lambda}\right)$. Such representations are said to be of class one with respect to $H_{\lambda}$. Let $m_{\lambda, \delta}$ denote it multiplicity in $L^{2}\left(G_{\lambda} / H_{\lambda}\right)$. Equivalently, $m_{\lambda}$ is the dimensions of vectors in the representation space of $\delta$ that are invariant under the action of $H_{\lambda}$. Let $\mathfrak{H}_{\delta}$ denote the space of functions in $L^{2}\left(G_{\lambda} / H_{\lambda}\right)$ which transform according to $\delta$, and $P_{\lambda, \delta}$ the orthogonal projection operator from $L^{2}\left(G_{\lambda} / H_{\lambda}\right)$ onto $\mathfrak{H}_{\delta}$. Then

$$
f=\sum_{\delta \in \Delta_{\lambda}} P_{\lambda, \delta} f
$$

is the primary decomposition of functions in $L^{2}\left(G_{\lambda} / H_{\lambda}\right)$, where $\Delta_{\lambda}$ denotes the set of all $H_{\lambda}$ class one representations. Of course we could further decompose $H_{\delta}$ into $m_{\lambda, \delta}$ irreducible pieces, but in general there is no canonical way of doing this. Let $K_{\lambda, \delta}\left(g_{\lambda}\right)$ denote the bi- $H_{\lambda}$-invariant kernel on $G_{\lambda}$ that realizes the projection operator $P_{\lambda, \delta}$ as a convolution operator on $G_{\lambda}$,

$$
P_{\lambda, \delta} \psi\left(g_{\lambda}^{\prime}\right)=\int_{G_{\lambda}} \psi\left(g_{\lambda}^{\prime} g_{\lambda}^{-1}\right) K_{\lambda, \delta}\left(g_{\lambda}\right) d g_{\lambda}
$$


for $g_{\lambda}^{\prime} \in G_{\lambda}$ and functions $\psi$ on $G_{\lambda}$ satisfying $\psi\left(g_{\lambda}^{\prime} h_{\lambda}\right)=\psi\left(g_{\lambda}^{\prime}\right)$.

Now for each fixed $g \in G$ apply (2.8) and (2.9) at $g_{\lambda}^{\prime}=e$ to the function $\psi\left(g_{\lambda}^{\prime}\right)=\phi_{\lambda}\left(g g_{\lambda}^{\prime}\right)$ and substitute into (2.6) to obtain

$$
F_{\lambda}(g, x)=\sum_{\delta \in \Delta_{\lambda}} F_{\lambda, \delta}(g, x)
$$

where

$$
F_{\lambda, \delta}(g, x)=\int_{H} \int_{G_{\lambda}} \phi_{\lambda}\left(g h g_{\lambda}^{-1}\right) K_{\lambda, \delta}\left(g_{\lambda}\right) e^{i\left\langle x, \rho(g h) z_{\lambda}\right\rangle} d g_{\lambda} d h .
$$

Now (2.3) and (2.10) give the final Fourier decomposition formula

$$
f(g, x)=\int_{\Lambda} \sum_{\delta \in \Delta_{\lambda}} F_{\lambda, \delta}(g, x) d \nu(\lambda)
$$

Substituting the definition of $\phi_{\lambda}$ and (2.2) in (2.11) yields

$$
\begin{aligned}
F_{\lambda, \delta}(g, x)=(2 \pi)^{-m} \int_{H} \int_{G_{\lambda}} \int_{W^{\perp}} & f\left(g h g_{\lambda}^{-1}, \rho\left(g h g_{\lambda}^{-1}\right)(0, z)\right) \\
\times & K_{\lambda, \delta}\left(g_{\lambda}\right) e^{-i\left\langle z, z_{\lambda}\right\rangle} e^{i\left\langle x, \rho(g h) z_{\lambda}\right\rangle} d z d g_{\lambda} d h
\end{aligned}
$$

which expresses the Fourier components $F_{\lambda, \delta}$ explicitly in terms of $f$. It is more illuminating, however, to introduce the functions $\phi_{\lambda, \delta}$ on $G / H_{\lambda}$ by

$$
\phi_{\lambda, \delta}(g)=\int_{G_{\lambda}} \phi_{\lambda}\left(g g_{\lambda}^{-1}\right) K_{\lambda, \delta}\left(g_{\lambda}\right) d g_{\lambda} .
$$

These functions play the role of the "Fourier transform" of $f$ and are given explicitly in terms of $f$ by

$$
\phi_{\lambda, \delta}(g)=(2 \pi)^{-m} \int_{G_{\lambda}} \int_{W^{\perp}} f\left(g g_{\lambda}^{-1}, \rho\left(g g_{\lambda}^{-1}\right)(0, z)\right) e^{-i\left\langle z, z_{\lambda}\right\rangle} K_{\lambda, \delta}\left(g_{\lambda}\right) d z d g_{\lambda}
$$

while the Fourier components are given in terms of the Fourier transform by

$$
F_{\lambda, \delta}(g, x)=\int_{H} \phi_{\lambda, \delta}(g h) e^{i\left\langle x, \rho(g h) z_{\lambda}\right\rangle} d h .
$$

By the orthogonality of the decomposition (2.8) we obtain the Plancherel formula

$$
\|f\|_{2}^{2}=(2 \pi)^{m} \int_{\Lambda} \sum_{\delta \in \Delta_{\lambda}}\left\|\phi_{\lambda, \delta}\right\|_{2}^{2} d \nu(\lambda)
$$

Notice that $\phi_{\lambda, \delta}$ is a function on $G / H_{\lambda}$ with the property

$$
\phi_{\lambda, \delta}\left(g g_{\lambda}\right) \in H_{\delta}
$$

as a function of $g_{\lambda}$ for each fixed $g \in G$, and also the action of $G \times V$ on $f$ intertwines the action (2.7) on $\phi_{\lambda, \delta}$. Therefore, the space of all functions $F_{\lambda, \delta}$ given by (2.16) under the action of $G \times V$ realizes a primary representation of $G \times V$ consisting of $m_{\lambda, \delta}$ copies of the irreducible representation in Mackey's theory associated to the orbit $X_{\lambda}$ and representation $\delta$ of $G_{\lambda}$. Finally, the Fourier transform map $f \rightarrow \phi_{\lambda, \delta}$ is an isometry of $L^{2}(G \times V / H \times W)$ onto the $L^{2}$ space of all $\phi_{\lambda, \delta}$ satisfying (2.18) for which the right side of (2.17) is finite, since we have already observed that the mapping $f \rightarrow \phi_{\lambda}$ is onto and the decomposition $\phi_{\lambda} \rightarrow \phi_{\lambda, \delta}$ is onto by the theory of induced representations. 
We can summarize the results as follows:

THEOREM 2.1. Define a Fourier transform $f \rightarrow \phi_{\lambda, \delta}$ for $f \in L^{2}(G \times V / H \times W)$ by (2.15), the integral over $W^{\perp}$ interpreted as a suitable limit. Then $\phi_{\lambda, \delta}$ is a function on $G / H_{\lambda}$ satisfying (2.18) and such that the right side of (2.17) is finite, and the mapping $f \rightarrow \phi_{\lambda, \delta}$ is an isometry onto the space of all such functions. Define the Fourier components $F_{\lambda, \delta}$ by (2.16). Then $f$ is a direct integral of Fourier components given by (2.12), the integral being interpreted in a suitable limiting sense, and this constitutes a decomposition of $L^{2}(G \times V / H \times W)$ as a representation of $G \times V$ into a direct integral of primary representation. Each component $F_{\lambda, \delta}$ lies in a space of $m_{\lambda, \delta}$ copies of the irreducible representation associated to the orbit $X_{\lambda}$ and the representation $\delta$ of $G_{\lambda}$.

REMARK. The theorem is most useful in the case that all multiplicites $m_{\lambda, \delta}$ are one, for then the primary representations are irreducible. One case in which this is always true is if $G \times V / H \times W$ is an affine symmetric space, in the sense that there exists an involution $\sigma$ of $G \times V$ such that $H \times W$ is the fixed-point set of $\sigma$. It is easy to see that $\sigma$ must then preserve $G_{\lambda}$ so that $G_{\lambda} / H_{\lambda}$ is a compact symmetric space, and it is well known that the irreducible represenations of $G_{\lambda}$ occur with multiplicity one in $L^{2}\left(G_{\lambda} / H_{\lambda}\right)$.

If $T$ is a bounded operator on $L^{2}(G \times V / H \times W)$ which commutes with the group action, then there exists a bounded multiplier $\psi(\lambda, \delta)$ such that

$$
T f(g, x)=\int_{\Lambda} \sum_{\delta \in \Delta_{\lambda}} \psi(\lambda, \delta) F_{\lambda, \delta}(g, x) d \nu(\lambda)
$$

if $f$ is given by $(2.13)$, or

$$
T F_{\lambda, \delta}=\psi(\lambda, \delta) F_{\lambda, \delta}
$$

if $T F_{\lambda, \delta}$ is well defined (the functions $F_{\lambda, \delta}$ are not $L^{2}$ ). In order to compute the multiplier $\psi(\lambda, \delta)$ it is convenient to have an explicit expression for one particular function $F_{\lambda, \delta}$ for each $\lambda, \delta$. Notice that (2.16) gives such an expression in terms of $\phi_{\lambda, \delta}$, once we find $\phi_{\lambda, \delta}$ satisfying (2.18); however, neither (2.14) nor (2.15) give a satisfactory expression for $\phi_{\lambda, \delta}$. In the next section we will write down more explicit expressions in some special cases.

3. Grassmannian bundles. We now specialize to the case of the Grassmannian bundle $P_{n, k}$ of affine $k$-planes in $\mathbf{R}^{n}$ where $k \leq n-1$. Here we may take $G=S O(n)$ and $\rho$ is the standard representation of $G$ on $V=\mathbf{R}^{n}$. Let $\pi_{0}$ denote the $k$-plane through the origin spanned by the first $k$ basis vectors. Then the isotropy subgroup of $G \times V$ fixing $\pi_{0}$ is $H \times W$, where $H=S(O(k) \times O(n-k))$ consists of matrices of determinant one in block diagonal form $\left(\begin{array}{ll}a & 0 \\ 0 & b\end{array}\right)$ with $a \in O(k)$ and $b \in O(n-k)$, and $W$ is the $k$-dimensional subspace of vectors of the form $\left(\begin{array}{l}y \\ 0\end{array}\right)$ with $y \in \mathbf{R}^{k}$. We could equally well take $G=O(n)$, in which case $H=O(k) \times O(n-k)$. The only change would be that in the case $n=2 k+1$, pairs of representations will coalesce into one. In either case, $G \times V / H \times W$ is an affine symmetric space in that $H \times W$ is the fixed-point set of the involution

$$
\sigma\left(\left(\begin{array}{ll}
a & b \\
c & b
\end{array}\right) \times\left(\begin{array}{l}
y \\
z
\end{array}\right)\right)=\left(\begin{array}{rr}
a & -b \\
-c & d
\end{array}\right) \times\left(\begin{array}{c}
y \\
-z
\end{array}\right)
$$


Now the orbits in $W^{\perp}$ under the action of $H$ are exactly the spheres of various radii and the origin, but we may ignore the origin since it is a set of measure zero. Thus it is natural to take the parameter set $\lambda$ to be the positive reals, and

$$
z_{\lambda}=\left(\begin{array}{c}
0 \\
\vdots \\
0 \\
\lambda
\end{array}\right) \quad \text { for each } \lambda>0 .
$$

In this way all the groups $H_{\lambda}$ are the same, namely $S(O(k) \times O(n-k-1))$, in the form $\left(\begin{array}{ll}a & 0 \\ 0 & b\end{array}\right)$ as above with $b=\left(\begin{array}{ll}c & 0 \\ 0 & 1\end{array}\right)$ and $c \in O(n-k-1)$. Similarly all the $G_{\lambda}$ groups can be identified with $S O(n-1)$ in the form $\left(\begin{array}{ll}d & 0 \\ 0 & 1\end{array}\right)$ with $d \in S O(n-1)$.

Next we need to identify those representations of $G_{\lambda}$ which are of class one with respect to $H_{\lambda}$. Since $G_{\lambda} / H_{\lambda}$ is the compact symmetric space $G_{n-1, k}$, the answer is well known. Choose the maximal torus $T^{\nu}$ in $G_{\lambda}$ consisting of rotations in the $1-2,3-4, \ldots,(2 \nu-1)-2 \nu$ planes, where $\nu=[(n-1) / 2]$. Irreducible representations of $G_{\lambda}$ are indexed by highest weight vectors $\delta=\left(\delta_{1}, \ldots, \delta_{\nu}\right)$ with respect to $T^{\nu}$, where $\delta_{1}, \ldots, \delta_{\nu}$ are integers satisfying $\delta_{1} \geq \delta_{2} \geq \cdots \geq \delta_{\nu} \geq 0$ if $n-1$ is odd and $\delta_{1} \geq \delta_{2} \geq \cdots \geq\left|\delta_{\nu}\right|$ if $n-1$ is even. The weight vectors $\delta$ corresponding to class one representations (see $\S 6$ ) are those satisfying

$$
\text { all } \delta_{j} \text { are even and } \delta_{j}=0 \text { if } j>k \text { or } j>n-k-1 \text {. }
$$

For each such $\delta$ our goal is to find an explicit expression for a function $\phi_{\lambda, \delta}$ satisfying the transformation law (2.18). To do this it is convenient to consider the Stiefel manifolds which lie between the rotation group and the Grassmannian manifolds. Let $S_{m}^{n}$ denote the set of $n \times m$ real matrices $\omega=\left(\omega_{1}, \ldots, \omega_{m}\right)$ whose columns $\omega_{j}$ are orthonormal vectors in $\mathbf{R}^{n}$. Equivalently, $\omega^{\operatorname{tr}} \omega=I_{m \times m}$. Then $S_{m}^{n}=S O(n) / S O(n-m)$ for $m \leq n-1$. Notice that $O(m)$ acts on $S_{m}^{n}$ by multiplication on the right and the quotient is exactly $G_{n, k}$ for $k=n-m$. Thus functions on $G_{n, k}$ may be thought of as functions on $S_{m}^{n}$ which are invariant under right multiplication by $O(m)$. We need a slight extension of this idea to understand condition (2.18).

Now fix the values of $k$ and $m$ with $k+m=n$ and $1 \leq k, m \leq n-1$. For $\omega \in S_{m}^{n}$ write $\omega=\left(\omega^{\prime}, \omega_{m}\right)$, where $\omega^{\prime}=\left(\omega_{1}, \ldots, \omega_{m-1}\right)$. We think of $\omega^{\prime}$ as an element of $S_{m-1}^{n-1}$ for the Euclidean space $\omega_{m}^{\perp}$. Let us write $\varkappa_{\delta}\left(\omega_{m}^{\perp}\right)$ to denote the space of functions on $S_{m-1}^{n-1}\left(\omega_{m}^{\perp}\right)$ which transform under the left action of $S O(n-1)$ on $S_{m-1}^{n-1}\left(\omega_{m}^{\perp}\right)$ according to the representation $\delta$.

LEMMA 3.1. There is a one-to-one correspondence (G-equivariant) between functions $\phi$ on $G$ satisfying

$$
\begin{cases}\phi(g)=\phi\left(g h_{\lambda}\right) & \text { for all } h_{\lambda} \in H_{\lambda}, \\ \phi\left(g g_{\lambda}\right) \in H_{\delta} & \text { as a function of } g_{\lambda} \in G_{\lambda} \text { for all } g \in G\end{cases}
$$

and functions $f$ on $S_{m}^{n}$ satisfying

$$
\begin{cases}f\left(\omega^{\prime}, \omega_{m}\right)=f\left(\omega^{\prime} r, \omega_{m}\right) & \text { for all } r \in O(m-1) \text { and } \\ f\left(\omega^{\prime}, \omega_{m}\right) \in H_{\delta}\left(\omega_{m}^{\perp}\right) & \text { for each fixed unit vector } \omega_{m} \in \mathbf{R}^{n},\end{cases}
$$


the correspondence being given by

$$
\phi(g)=f(\omega)
$$

if $\omega$ equals the last $m$ columns of $g$.

ProOF. Note that (3.4) is the same as $\phi(g)=f\left(g\left(\begin{array}{c}0 \\ I_{m}\end{array}\right)\right)$. From this it is easy to see the equivalence of the conditions $\phi(g)=\phi\left(g h_{\lambda}\right)$ and $f\left(\omega^{\prime}, \omega_{m}\right)=f\left(\omega^{\prime} r, \omega_{m}\right)$ and that the mapping is well defined and $G$-equivariant. For the case $g=I$ it is clear that $\phi\left(g g_{\lambda}\right) \in H_{\delta}$ is equivalent to $f\left(\omega^{\prime}, \omega_{m}\right) \in H_{\delta}\left(\omega_{m}^{\perp}\right)$ for

$$
\omega_{m}=\left(\begin{array}{c}
0 \\
\vdots \\
0 \\
1
\end{array}\right) \text {, }
$$

and the general case follows by $G$-equivariance.

The problem now is to find functions satisfying (3.3). To simplify matters we will first drop the invariance condition $f\left(\omega^{\prime}, \omega_{m}\right)=f\left(\omega^{\prime} r, \omega_{m}\right)$, for we can obtain it in the end by an averaging process. Now in [10] we gave a formula for some functions in $\mathcal{H}_{\delta}\left(\omega_{m}^{\perp}\right)$. For simplicity first take

$$
\omega_{m}=\left(\begin{array}{c}
0 \\
\vdots \\
0 \\
1
\end{array}\right) .
$$

Let $a_{j}=e_{2 j-1}+i e_{2 j}$ denote the vector in $\mathbf{C}^{n}$ with the value 1 in the $2 j-1$ position and $i$ in the $2 j$ position for $j=1, \ldots, \nu$ (these are clearly positive root vectors for the maximal torus $\left.T^{\nu}\right)$. Let $M_{j}\left(\omega^{\prime}\right)$ denote the determinant of the $j \times j$ matrix $\left\{a_{p} \cdot \omega_{m-q}\right\}, p, q=1, \ldots, j$, for $j \leq \nu_{0}$ where $\nu_{0}=\min (m-1, k)$. If $n-1$ is even and $m-1=k=\nu$ we will also need to define $M_{\nu}^{-}\left(\omega^{\prime}\right)$ in the same way except that $a_{\nu}$ is replaced by $\bar{a}_{\nu}$.

Let $\delta=\left(\delta_{1}, \ldots, \delta_{\nu_{0}}, 0, \ldots, 0\right)$ be a weight satisfying (3.1). Then Theorem 1 of [10] says that

$$
f\left(\omega^{\prime}\right)=\prod_{j=1}^{\nu_{0}} M_{j}\left(\omega^{\prime}\right)^{2 \lambda_{j}}
$$

is in $\varkappa_{\delta}\left(\omega_{m}^{\perp}\right)$ for $2 \lambda_{j}=\delta_{j}-\delta_{j+1}$ (in the special case $\nu=\nu_{0}=m-1=k$ if $\delta_{\nu}$ is negative we replace $M_{\nu}\left(\omega^{\prime}\right)$ by $M_{\nu}^{-}\left(\omega^{\prime}\right)$ and $\left.2 \lambda_{\nu}=-\delta_{\nu}\right)$.

Now we want to understand the case of more general $\omega_{m}$, and for this it is necessary only to abstract the salient features of the vectors $a_{j}$. It is clear that we have $\nu$ linearly independent vectors in $\mathbf{C}^{n}$ satisfying

$$
a_{i} \cdot a_{j}=0 \text { and } a_{j} \cdot \omega_{m}=0
$$

and conversely if (3.6) holds then (3.5) is in $\mathcal{H}_{\delta}\left(\omega_{m}^{\perp}\right)$. (In the case $\nu=\nu_{0}=m-1=k$ we have to distinguish the two orientations of the vectors $a_{j}$ in order to decide the sign of $\delta_{\nu}$.) To make the dependence on the vectors $a_{j}$ clear we will write $M_{j}\left(\omega^{\prime}, a\right)$. 
Now to construct functions satisfying (3.3) we need to solve the following problem: Find $f\left(\omega^{\prime}, \omega_{m}\right)$ such that for each fixed $\omega_{m}$ there exist vectors $a_{j}\left(\omega_{m}\right)$ such that $f\left(\omega^{\prime}, \omega_{m}\right)=M_{j}\left(\omega^{\prime}, a\left(\omega_{m}\right)\right)$. While it is obvious that such functions exist, we would like a solution with as simple an expression as possible. It turns out that there is a very elegant solution, which we have discovered by trial and error and other methods and which is easily verified to have the desired properties. The basic recipe is to let $\tilde{M}_{j}(\omega)$ be the determinant of the $j \times j$ matrix $\left\{a_{p} \cdot \omega_{m-q+1}\right\}, p, q=1, \ldots, j$ for $2 \leq j \leq \nu_{0}+1$, where the vectors $a_{j}=e_{2 j-1}+i e_{2 j}$ as before. This definition makes sense in all cases except when $n$ is odd, $m-1=k=\nu$, and $j=\nu+1$. In that case we define $\tilde{M}_{m}^{ \pm}(\omega)$ to be the determinant of

$$
\left(\begin{array}{cccc}
a_{1} \cdot \omega_{m} & a_{1} \cdot \omega_{m-1} & \cdots & a_{1} \cdot \omega_{1} \\
\vdots & \vdots & & \vdots \\
a_{m-1} \cdot \omega_{m} & a_{m-1} \cdot \omega_{m-1} & \cdots & a_{m-1} \cdot \omega_{1} \\
e_{n} \cdot \omega_{m} \pm 1 & e_{n} \cdot \omega_{m-1} & \cdots & e_{n} \cdot \omega_{1}
\end{array}\right)
$$

LEMMA 3.2. (a) In all cases except when $n$ is odd and $m-1=k=\nu$, let

$$
a_{j}\left(\omega_{m}\right)=\left(\omega_{m} \cdot a_{1}\right) a_{j+1}-\left(\omega_{m} \cdot a_{j+1}\right) a_{1} .
$$

Then for each fixed $\omega_{m}$ with $\omega_{m} \cdot a_{1} \neq 0$ the vectors $a_{j}\left(\omega_{m}\right)$ satisfy (3.6) and

$$
\tilde{M}_{j}(\omega)=c_{j}\left(\omega_{m}\right) M_{j-1}\left(\omega^{\prime}, a\left(\omega_{m}\right)\right)
$$

for some constant $c_{j}\left(\omega_{m}\right)$.

(b) When $n$ is odd and $m-1=k=\nu$, define $a_{j}\left(\omega_{m}\right)$ by (3.7) for $1 \leq j \leq m-1$ and

$$
a_{m}\left(\omega_{m}\right)=\left(\omega_{m} \cdot a_{1}\right)\left(e_{n} \pm \omega_{m}\right)-\left(\omega_{m} \cdot\left(e_{n} \pm \omega_{m}\right)\right) a_{1} .
$$

Then again (3.6) and (3.8) hold, with the orientation of the vectors $a_{j}\left(\omega_{m}\right)$ agreeing with the choice of \pm .

PROOF. For (a) take the matrix

$$
\left(\begin{array}{ccc}
a_{1} \cdot \omega_{m} & \cdots & a_{1} \cdot \omega_{m-j+1} \\
\vdots & & \vdots \\
a_{j} \cdot \omega_{m} & \cdots & a_{j} \cdot \omega_{m-j+1}
\end{array}\right)
$$

whose determinant is $\tilde{M}_{j}(\omega)$, multiply all the rows except the first by $a_{1} \cdot \omega_{m}$, and then subtract $a_{1} \cdot \omega_{i}$ times the first row from the $i$ th row. This only changes the determinant by the factor $\left(a_{1} \cdot \omega_{m}\right)^{j-1}$, and clears the first column to

$$
\left(\begin{array}{c}
a_{1} \cdot \omega \\
0 \\
\vdots \\
0
\end{array}\right)
$$

so we can read off (3.8). Also (3.6) for $a_{j}\left(\omega_{m}\right)$ is an immediate consequence of definition (3.7) and the analogous properties of the $a_{j}$. For (b) we do an analogous procedure to the matrix whose determinant is $\tilde{M}_{m}^{ \pm}(\omega)$. Again we multiply each row 
except the first by $a_{1} \cdot \omega_{m}$, and subtract $a_{1} \cdot \omega_{i}$ times the first row from the $i$ th row for $2 \leq i \leq m-1$, and $e_{n} \cdot \omega_{n} \pm 1$ times the first row from the last row. This clears the first column as before, but we require one additional observation. The last row is now $\left(0 d_{m} \cdot \omega_{m-1} \cdots d_{m} \cdot \omega_{1}\right)$, where $d_{m}=\left(a_{1} \cdot \omega_{m}\right) e_{n}-\left(e_{n} \cdot \omega_{m} \pm 1\right) a_{1}$. The observation is that while $d_{m}$ is not equal to $a_{m}\left(\omega_{m}\right)$ as given by (3.9), we still have $d_{m} \cdot \omega_{i}=a_{m}\left(\omega_{m}\right) \cdot \omega_{i}$ for $i \leq m-1$. The reason for this is simply that $\omega_{m} \cdot \omega_{i}=0$ since $\omega \in S_{m}^{n}$. The verification of (3.6) for $i, j \leq m-1$ is immediate, and

$$
\begin{aligned}
a_{m}\left(\omega_{m}\right) \cdot a_{m}\left(\omega_{m}\right)= & \left(\omega_{m} \cdot a_{1}\right)^{2}\left(e_{n} \pm \omega_{m}\right) \cdot\left(e_{n} \pm \omega_{m}\right) \\
& -2\left(\omega_{m} \cdot a_{1}\right)\left(\omega_{m} \cdot e_{n} \pm 1\right) a_{1} \cdot\left( \pm \omega_{m}\right) \\
= & \left(\omega_{m} \cdot a_{1}\right)^{2}\left(2 \pm 2 e_{n} \cdot \omega_{m}-2\left(1 \pm \omega_{m} \cdot e_{n}\right)\right)=0 .
\end{aligned}
$$

A simpler computation shows $a_{j}\left(\omega_{m}\right) \cdot a_{m}\left(\omega_{n}\right)=0$ for $j<m$.

Finally, we need to consider the relative orientations of the vectors $a_{j}\left(\omega_{m}\right)$. Since the orientation clearly varies continuously over the set of $\omega_{m}$ for which $\tilde{M}_{m}^{ \pm}(\omega)$ is not identically zero in $\omega^{\prime}$, it is necessary to observe that this set is connected, and then compute the orientation for one choice of $\omega_{m}$. Now the argument just given shows that $\tilde{M}_{m}^{ \pm}(\omega)$ is not identically zero if $a_{1} \cdot \omega_{m} \neq 0$, and analogous reasoning using the $i$ th row in place of the first row shows that $\tilde{M}_{m}^{ \pm}(\omega)$ is not identically zero if $a_{i} \cdot \omega_{m} \neq 0$ for one $i, 1 \leq i \leq m$. This leaves only two possibilities, $\omega_{m}= \pm e_{n}$, of which one occurs (when the \pm signs are mismatched). This shows that the set of $\omega_{m}$ for which $\tilde{M}_{m}^{ \pm}(\omega) \not \equiv 0$ to be the sphere minus a point, which is connected. Choosing $\omega_{m}=e_{n}$ we also see $\tilde{M}_{m}^{+}(\omega)=2(-1)^{m} M_{m-1}^{+}\left(\omega^{\prime}\right)$ which gives the positive orientation. Now for $\omega_{m}=-e_{n}$ we have $\tilde{M}_{m}^{-}(\omega)=2(-1)^{m-1} M_{m-1}^{+}\left(\omega^{\prime}\right)$, but this is now the negative orientation since it takes an orientation reversing map to send $-e_{n}$ to $e_{n}$ and to leave the other basis elements fixed. Q.E.D. let

$$
f_{\delta}(\omega)=\int_{O(m-1)} \prod_{j=1}^{\nu_{0}} \tilde{M}_{j+1}\left(\omega^{\prime} r, \omega_{m}\right)^{2 \lambda_{j}} d r
$$

where

$$
2 \lambda_{j}=\delta_{j}-\delta_{j+1}
$$

(in the special case $\nu=\nu_{0}=m-1=k$ we take $2 \lambda_{\nu}=\left|\delta_{\nu}\right|$ and use $\tilde{M}_{m}^{+}$or $\tilde{M}_{m}^{-}$ according to $\operatorname{sgn} \delta_{\nu}$ ). Then $f_{\delta}$ satisfies (3.3) and is not identically zero.

ProOF. The first part of (3.3) follows from the $O(m-1)$ integral in (3.10), and the second part follows from Lemma 3.2 and the invariance of this condition under right multiplication $\omega^{\prime} \rightarrow \omega^{\prime} r$. To see that $f_{\delta}$ is not identically zero we choose $\omega_{n}=e_{1}, \omega_{m-1}=e_{3}, \ldots, \omega_{j}=e_{2 m-2 j+1}$. With this choice $a_{p} \cdot \omega_{q}$ is always real, so $\tilde{M}_{j+1}\left(\omega^{\prime} r, \omega_{m}\right)^{2 \lambda_{j}} \geq 0$, and we have in (3.10) an integral of a nonnegative function. The integrand is clearly continuous in $r$ and assumes the value one when $r$ is the identity, so $f_{\delta}$ is positive. Q.E.D.

For some applications it is useful to note that (3.10) produces a "marked" function. From the point of view of the group $G$, we have produced a vector in the representation induced from the representation $\pi_{\delta}$ of $G_{\lambda}$. Now the well-known 
branching law and the Frobenius reciprocity theorem give a description of this induced representation as a multiplicity one direct sum of representations $\pi_{\delta^{\prime}}$ of $G$, where the highest weights $\delta^{\prime}$ of $G$ satisfy the interlacing condition

$$
\begin{aligned}
& \delta_{1}^{\prime} \geq \delta_{1} \geq \delta_{2}^{\prime} \geq \cdots \geq \delta_{\nu}^{\prime} \geq\left|\delta_{\nu}\right|, \quad n \text { odd, or } \\
& \delta_{1}^{\prime} \geq \delta_{1} \geq \delta_{2}^{\prime} \geq \cdots \geq \delta_{\nu} \geq\left|\delta_{\nu+1}^{\prime}\right|, \quad n \text { even. }
\end{aligned}
$$

Here $T^{\nu}$ is a maximal torus for both $G_{\lambda}$ and $G$ if $n$ is odd, while for $n$ even we enlarge $T^{\nu}$ to include rotations in the $(n-1)-n$ plane to obtain a maximal torus for $G$. In all cases except $\nu=\nu_{0}=m-1=k$ with $\delta_{\nu} \neq 0$, it is clear from Theorem 1 of $[10]$ that $f_{\delta}$ is a highest weight vector for the representation $\pi_{\delta^{\prime}}$ of $G$ with $\delta^{\prime}=\left(\delta_{1}, \delta_{1}, \delta_{2}, \delta_{3}, \ldots, \delta_{\nu_{0}}, 0, \ldots, 0\right)$. Thus $f_{\delta}$ lies in a fixed one-dimensional space.

Finally we need to substitute the function $\phi$ corresponding to $f_{\delta}$ into (2.16) via Lemma 3.1 and to simplify the resulting explicit expression for a Fourier component $F_{\lambda, \delta}$ in each irreducible representation of $G \times V$. In particular, we can combine the $H$ integral in (2.16) (really an integral over $H / H_{\lambda}$ ) and the $O(m-1)$ integral in (3.10). To simplify notation set

$$
\tilde{M}_{\delta}(\omega)=\prod_{j=1}^{\nu_{0}} \tilde{M}_{j+1}(\omega)^{2 \lambda_{j}}
$$

so that

$$
f_{\delta}(\omega)=\int_{O(m-1)} \tilde{M}_{\delta}\left(\omega\left(\begin{array}{ll}
r & 0 \\
0 & 1
\end{array}\right)\right) d r .
$$

From (2.16) and Lemma 3.1 we have

$$
\begin{aligned}
F_{\lambda, \delta}(g, x) & =\int_{S(O(k) \times O(m))} \phi(g h) e^{i \lambda\left\langle x, g h e_{n}\right\rangle} d h \\
& =\int_{S(O(k) \times O(m))} f_{\delta}\left(g h\left(\begin{array}{c}
0 \\
I_{m}
\end{array}\right)\right) e^{i \lambda\left\langle x, g h e_{n}\right\rangle} d h \\
& =\int_{O(m)} f_{\delta}\left(g\left(\begin{array}{l}
0 \\
s
\end{array}\right)\right) e^{i \lambda\left\langle x, g\left(s e_{n}\right)\right\rangle} d s
\end{aligned}
$$

since the $O(k)$ component of $h$ does not change the integrand (here $\left(s e_{n}\right)$ means $\left.\left(\begin{array}{ll}I & 0 \\ 0 & s\end{array}\right) e_{n}\right)$. Now substitute (3.13) and make the change of variable $s \rightarrow s\left(\begin{array}{cc}r^{-1} & 0 \\ 0 & 1\end{array}\right)$. Notice that this does not change $s e_{n}$. This makes the integrand independent of $r$, so

$$
F_{\lambda, \delta}(g, x)=\int_{O(m)} \tilde{M}_{\delta}\left(g\left(\begin{array}{l}
0 \\
s
\end{array}\right)\right) e^{i \lambda\left\langle x, g\left(s e_{n}\right)\right\rangle} d s .
$$

Let us introduce some simplifying notation. A $k$-plane will be parametrized $[\omega, y]$ with $\omega \in S_{m}^{n}$ and $y \in \mathbf{R}^{m}$, so that $[\omega, y]$ is the set of points in $\mathbf{R}^{n}$ of the form $u+\omega y$, where $u \in \omega^{\perp}$ (i.e., $u^{\text {tr }} \omega=0$ ). The extent of nonuniqueness is that $\left[\omega s, s^{-1} y\right]=[\omega, y]$ for any $s \in O(m)$. The relationship with the coset $(g H, x+W)$ notation is that $\omega=g\left(\begin{array}{c}0 \\ I_{m}\end{array}\right)$ and $x \equiv \omega y \bmod W$.

Then $g\left(\begin{array}{l}0 \\ s\end{array}\right)=\omega s$ and $g\left(s e_{n}\right)=\omega s e_{m}$, where $e_{m}$ is the $m$ th unit vector in $\mathbf{R}^{m}$. Thus $\left\langle x, g\left(s e_{n}\right)\right\rangle=\left\langle\omega y, \omega s e_{m}\right\rangle$ and since $\omega \in S_{m}^{n}$ this is equal to $\left\langle y, s e_{m}\right\rangle$, where 
the inner product is now in $\mathbf{R}^{m}$. Thus (3.14) becomes

$$
F_{\lambda, \delta}([\omega, y])=\int_{O(m)} \tilde{M}_{\delta}(\omega s) e^{i \lambda\left\langle y, s e_{m}\right\rangle} d s .
$$

If $\nu_{0}<m$ then it suffices to integrate over $S_{\nu_{0}}^{m}$, since only $\nu_{0}$ columns of $\omega$ are needed to define $\tilde{M}_{\delta}$.

4. Harmonic analysis of induced representations. Now we consider the general case of a semidirect product $G \times V$, where $V$ is abelian, and the harmonic analysis of any representation induced from a subgroup $H \times W$. More specifically, we assume that $G$ and $V$ are locally compact groups, $V$ is abelian, and $\rho$ is a continuous homomorphism from $G$ into the automorphisms of $V$, so $G \times V$ is a locally compact group under the composition law

$$
\left(g_{1}, x_{1}\right) \circ(g, x)=\left(g_{1} g, x_{1}+\rho\left(g_{1}\right) x\right) .
$$

We assume $H$ and $W$ are closed subgroups of $G$ and $V$ respectively, with $\rho(H) W \subseteq$ $W$. Let $Z=V / W$, and let $\hat{V}, \hat{W}, \hat{Z}$ denote the respective dual groups of $V, W, Z$, so that $\hat{V}=\hat{W} \oplus \hat{Z}$ canonically. We assume the action $\rho(G)$ on $\hat{V}$ defined by $\rho(g) \xi(x)=$ $\xi\left(\rho(g)^{-1} x\right)$ is sufficiently regular for the Mackey theory [8] to be applicable and the same for the action of $\rho(H)$ on $\hat{W}$ and $\hat{Z}$. It suffices, for example, to assume that $G$ and $V$ are Lie groups.

Now by Mackey's theory, every irreducible unitary representation of $H \times W$ is itself an induced representation from a subgroup $H_{0} \times W$, so by "induction by stages" we can induce directly from $H_{0} \times W$ to $G \times V$. In other words, we may assume without loss of generality that the representation of $H \times W$ from which we are inducing has the form $\pi \times \chi$, where $\pi$ is an irreducible unitary representation of $H$ on $\mathscr{H}$, and $\chi \in \hat{W}$ such that $\rho(H) \chi=\chi$. The representation space is $L^{2}(G \times V, \mathcal{H}, \pi \times \chi)$, functions $f: G \rightarrow \not{H}$ satisfying

$$
f(g h, x+\rho(g) w)=\overline{\chi(w)} \pi(h)^{-1} f(g, x)
$$

and

$$
\int_{G / H} \int_{Z}\|f(g, \rho(g) z)\|^{2} d \bar{g} d z=\|f\|_{2}^{2}<\infty,
$$

where we have chosen (not canonically) a quasi-invariant measure $d \bar{g}$ on $G / H$ and an embedding of $Z$ in $V$, and $\|f(g, \rho(g) z)\|$ denotes the norm in $\mathcal{H}$. The induced representation action $T\left(g_{1}, x_{1}\right)$ in $L^{2}(G \times V, \not{H}, \pi \times \chi)$ is

$$
T\left(g_{1}, x_{1}\right) f(g, x)=J\left(g_{1}, g\right)^{1 / 2} f\left(g_{1} g, x_{1}+\rho\left(g_{1}\right) x\right),
$$

where $J\left(g_{1}, g\right)$ is the Radon-Nikodym derivative of the translate by $g_{1}$ of the measure $d \bar{g}$ with respect to $d \bar{g}$.

As before, the first step in the harmonic analysis of $T$ is to apply the abelian harmonic analysis on $Z$ to the function $f(g, \rho(g) z)$ for each fixed $g$. Thus

$$
\begin{aligned}
& \hat{f}(g, \zeta)=\int_{Z} f(g, \rho(g) z) \overline{\zeta(z)} d z \\
& f(g, \rho(g) z)=\int_{\hat{Z}} \hat{f}(g, \zeta) \zeta(z) d \zeta
\end{aligned}
$$




$$
\|f\|_{2}^{2}=\int_{G / H} \int_{\hat{Z}}\|\hat{f}(g, \zeta)\| d \zeta d \bar{g}
$$

if the Haar measures $d z$ and $d \varsigma$ are consistently normalized. From (4.1) and (4.4) we obtain

$$
f(g, x)=\int_{\hat{Z}} \overline{\chi\left(\rho(g)^{-1} x\right)} \hat{f}(g, \zeta) \varsigma\left(\rho(g)^{-1} x\right) d \zeta,
$$

where we have identified $\chi \in \hat{W}$ and $\varsigma \in \hat{Z}$ with elements of $\hat{V}$ under the canonical identification $\hat{V}=\hat{W} \oplus \hat{Z}$. (We denote the group product in $\hat{V}$ multiplicatively.)

Next we decompose $\hat{Z}$ into orbits $\hat{Z}_{\lambda}$ under $\rho(H)$, indexed by $\lambda \in \Lambda$, and choose $\zeta_{\lambda} \in \hat{Z}_{\lambda}$ for each $\lambda$. Let $H_{\lambda}$ denote the isotropy subgroup of $H$ for $\zeta_{\lambda}$. Now invoking the regularity assumption for the action of $\rho(H)$ on $\hat{Z}$, we can choose quasi-invariant measures $d \mu_{\lambda}$ on $H / H_{\lambda}$ and a measure $d \nu$ on $\Lambda$ such that

$$
\int_{\hat{Z}} \phi(\zeta) d \zeta=\int_{\Lambda}\left(\int_{H / H_{\lambda}} \phi\left(\rho(h)_{\zeta_{\lambda}}\right) d \mu_{\lambda}(\bar{h})\right) d \nu(\lambda)
$$

for any integrable function $\phi$ on $\hat{Z}$. We apply this to (4.6) to obtain

$$
f(g, x)=\int_{\Lambda} F_{\lambda}(g, x) d \nu(\lambda)
$$

where

$$
F_{\lambda}(g, x)=\int_{H / H_{\lambda}} \overline{\chi\left(\rho(g)^{-1} x\right)} \hat{f}\left(g, \rho(h) \zeta_{\lambda}\right) \rho(h)_{\zeta_{\lambda}}\left(\rho(g)^{-1} x\right) d \mu_{\lambda}(\hat{h}) .
$$

To simplify this we define

$$
\phi_{\lambda}(g)=\hat{f}\left(g, \varsigma_{\lambda}\right)
$$

From (4.1) and (4.3) we obtain

$$
\hat{f}\left(g, \rho(h) \zeta_{\lambda}\right)=\pi(h) \phi_{\lambda}(g h)
$$

and

$$
\phi_{\lambda}\left(g h_{\lambda}\right)=\pi\left(h_{\lambda}\right)^{-1} \phi_{\lambda}(g) \text { for all } h_{\lambda} \in H_{\lambda} .
$$

Then (4.8) becomes (using $\rho(h) \chi=\chi$ )

$$
F_{\lambda}(g, x)=\int_{H / H_{\lambda}}\left(x^{-1} \zeta_{\lambda}\right)\left(\rho(g h)^{-1} x\right) \pi(h) \phi_{\lambda}(g h) d \mu_{\lambda}(\bar{h}) .
$$

We also have

$$
\|f\|^{2}=\int_{G / H} \int_{\Lambda} \int_{H / H_{\lambda}}\left\|\phi_{\lambda}(g h)\right\|^{2} d \mu_{\lambda}(\bar{h}) d \nu(\lambda) d \bar{g}
$$

and we can combine the integrals over $G / H$ and $H / H_{\lambda}$ into a single integral over $G / H_{\lambda}$ with respect to a suitable quasi-invariant measure $d \mu_{\lambda}(\bar{g})$, so

$$
\|f\|^{2}=\int_{\Lambda} \int_{G / H_{\lambda}}\left\|\phi_{\lambda}(g)\right\|^{2} d \mu_{\lambda}(\bar{g}) d \nu(\lambda) .
$$

The next step, one that does not occur when $\pi$ is trivial, is to restrict $\pi$ to $\bar{H}_{\lambda}$ and decompose it into a direct integral of primary (or irreducible) representations. 
We assume that $\nvdash=\int_{A} \nvdash_{\alpha} d \alpha, \pi=\int_{A} \pi_{\alpha} d \alpha$ on $H_{\lambda}$ is such a decomposition, where $d \alpha$ is a suitable measure on an index set $A$ and for each $\alpha \in A, \pi_{\alpha}$ is a primary (or irreducible) unitary representation of $H_{\lambda}$ on the Hilbert space $\nvdash_{\alpha}$. We let \|\|$_{\alpha}$ denote the Hilbert space norm on $\nVdash_{\alpha}$, and let $P_{\alpha}$ denote the generalized projection operator on $\mathscr{H}$ to $\mathscr{H}_{\alpha}$ (unless $\mathscr{H}$ occurs discretely, it is not a subspace of $\mathscr{H}$ ). Then for any $\phi \in \mathscr{H}$,

$$
\phi=\int_{A} P_{\alpha} \phi d \alpha
$$

and

$$
\|\phi\|^{2}=\int_{A}\left\|P_{\alpha} \phi\right\|_{\alpha}^{2} d \alpha
$$

and we have

$$
P_{\alpha} \pi\left(h_{\lambda}\right) \phi=\pi_{\alpha}\left(h_{\lambda}\right) P_{\alpha} \phi \quad \text { for all } h_{\lambda} \in H_{\lambda} .
$$

Now we apply (4.14) to $\phi_{\lambda}(g h)$ and substitute into (4.12). We obtain

$$
F_{\lambda}(g, x)=\int_{A} F_{\lambda, \alpha}(g, x) d \alpha
$$

where

$$
F_{\lambda, \alpha}(g, x)=\int_{H / H_{\lambda}}\left(\chi^{-1} \zeta_{\lambda}\right)\left(\rho(g h)^{-1} x\right) \pi_{\alpha}(h) \phi_{\lambda, \alpha}(g h) d \mu_{\lambda}(\bar{h})
$$

and

$$
\phi_{\lambda, \alpha}(g)=P_{\alpha} \phi_{\lambda}(g) .
$$

Notice that $\phi_{\lambda, \alpha}: G \rightarrow \mathscr{H}_{\alpha}$ and

$$
\phi_{\lambda, \alpha}\left(g h_{\lambda}\right)=\pi_{\alpha}\left(h_{\lambda}\right)^{-1} \phi_{\lambda, \alpha}(g) \text { for all } h_{\lambda} \in H_{\lambda}
$$

in view of (4.11) and (4.16). Also from (4.13) and (4.15) we obtain

$$
\|f\|^{2}=\int_{\Lambda} \int_{A} \int_{G / H_{\lambda}}\left\|\phi_{\lambda, \alpha}(g)\right\|_{\alpha}^{2} d \mu_{\lambda}(\bar{g}) d \alpha d \nu(\lambda)
$$

which shows that for a.e. choice of $\lambda$ and $\alpha, \phi_{\lambda, \alpha} \in L^{2}\left(G, H_{\lambda}, \pi_{\alpha}\right)$, which is the representation space for the representation of $G$ induced from $\pi_{\alpha}$ on $H_{\lambda}$. We may interpret (4.18) as defining an intertwining operator from $L^{2}\left(G, H_{\lambda}, \pi_{\alpha}\right)$ to a space of functions $F_{\lambda, \alpha}(g, x)$ on $G \times V$. In fact, taking the $T\left(g_{1}, x_{1}\right)$ action on $F_{\lambda, \alpha}$ given by (4.2), under (4.18) this is intertwined with

$$
T_{\lambda, \alpha}\left(g_{1}, x_{1}\right) \phi_{\lambda, \alpha}(g)=J\left(g_{1}, g\right)^{1 / 2}\left(\chi^{-1} \zeta_{\lambda}\right)\left(\rho\left(g g_{1}\right)^{-1} x_{1}\right) \phi_{\lambda, \alpha}\left(g_{1} g\right)
$$

and $J\left(g_{1}, g\right)$ is the correct Radon-Nikodym derivative for the measure $d \mu_{\lambda}(\bar{g})$ on $G / H_{\lambda}$. Thus the action of $G$ on $\phi_{\lambda, \alpha}$ is the induced representation from $\pi_{\alpha}$ on $H_{\lambda}$.

The last step is to decompose this induced representation by inducing to an intermediate group $G_{\lambda}$, the isotropy group of $\zeta_{\lambda}$ in $G$. Suppose

$$
\operatorname{ind}_{H_{\lambda} \uparrow G_{\lambda}} \pi_{\alpha}=\int_{\Delta_{\lambda, \alpha}} \pi_{\lambda, \alpha, \delta} d \delta
$$


is a direct integral decomposition into primary (or irreducible) representations of $G_{\lambda}$, and denote by $P_{\lambda, \alpha, \delta}$ the associated projection operator from $L^{2}\left(G_{\lambda}, H_{\lambda}, \pi_{\alpha}\right)$ onto the representation space for $\pi_{\lambda, \alpha, \delta}$. This means

$$
\begin{gathered}
\phi=\int_{\Delta_{\lambda, \alpha}} P_{\lambda, \alpha, \delta} \phi d \delta \\
\|\phi\|_{\lambda, \alpha}^{2}=\int_{\Delta_{\lambda, \alpha}}\left\|P_{\lambda, \alpha, \delta} \phi\right\|_{\lambda, \alpha, \delta}^{2} d \delta
\end{gathered}
$$

for any $\phi \in L^{2}\left(G_{\lambda}, H_{\lambda}, \pi_{\alpha}\right)$, where \|\|$_{\lambda, \alpha}$ denotes the norm on $L^{2}\left(G_{\lambda}, H_{\lambda}, \pi_{\alpha}\right)$ and \|\|$_{\lambda, \alpha, \delta}$ denotes the norm on the representation space for $\pi_{\lambda, \alpha, \delta}$. Also we have

$$
P_{\lambda, \alpha, \delta} \phi\left(g_{\lambda} g\right)=\pi_{\lambda, \alpha, \delta}\left(g_{\lambda}\right) P_{\lambda, \alpha, \delta} \phi(g)
$$

for any $g_{\lambda} \in G_{\lambda}$. Usually it is possible to realize the representation space of $\pi_{\lambda, \alpha, \delta}$ as a generalized subspace of $L^{2}\left(G_{\lambda}, H_{\lambda}, \pi_{\alpha}\right)$, in other words as functions on $G_{\lambda}$ satisfying (4.20), and $P_{\lambda, \alpha, \delta}$ as a convolution operator

$$
P_{\lambda, \alpha, \delta} \psi(g)=\int_{G_{\lambda}} \psi\left(g g_{\lambda}^{-1}\right) K_{\lambda, \alpha, \delta}\left(g_{\lambda}\right) d g_{\lambda} \quad\left(\text { for } g \in G_{\lambda}\right)
$$

with a suitable kernel $K_{\lambda, \alpha, \delta}$. We will adopt this notation in general.

Now we define (for $g \in G$ )

$$
\phi_{\lambda, \alpha, \delta}(g)=P_{\lambda, \alpha, \delta} \phi_{\lambda, \alpha}(g)=\int_{G_{\lambda}} \phi_{\lambda, \alpha}\left(g g_{\lambda}^{-1}\right) K_{\lambda, \alpha, \delta}\left(g_{\lambda}\right) d g_{\lambda} .
$$

This will be the final "Fourier transform," and it is given in terms of $f$ by

$$
\phi_{\lambda, \alpha, \delta}(g)=\int_{G_{\lambda}} \int_{Z} P_{\alpha} f\left(g g_{\lambda}^{-1}, \rho\left(g g_{\lambda}^{-1}\right) z\right) \overline{\zeta_{\lambda}(z)} K_{\lambda, \alpha, \delta}\left(g_{\lambda}\right) d z d g_{\lambda} .
$$

The associated Fourier component $F_{\lambda, \alpha, \delta}$ is

$$
F_{\lambda, \alpha, \delta}(g, x)=\int_{H / H_{\lambda}}\left(\chi^{-1} \varsigma_{\lambda}\right)\left(\rho(g h)^{-1} x\right) \pi_{\alpha}(h) \phi_{\lambda, \alpha, \delta}(g h) d \mu_{\lambda}(\bar{h}),
$$

the Fourier inversion formula is

$$
f(g, x)=\int_{\Lambda} \int_{A} \int_{\Delta_{\lambda, \alpha}} F_{\lambda, \alpha, \delta}(g, x) d \delta d \alpha d \nu(\lambda),
$$

and the Plancherel formula is

$$
\|f\|^{2}=\int_{\Lambda} \int_{A} \int_{\Delta_{\lambda, \alpha}} \| \phi_{\lambda, \alpha, \delta}^{2} d \delta d \alpha d \nu(\lambda)
$$

The action $T_{\lambda, \alpha}\left(g_{1}, x_{1}\right)$ on $\phi_{\lambda, \alpha, \delta}$ given by (4.22) intertwines the action $T\left(g_{1}, x_{1}\right)$ on $F_{\lambda, \alpha, \delta}$ given by (4.2), and so $F_{\lambda, \alpha, \delta}$ realizes the primary or irreducible representation of $G \times V$ associated to the point $\chi^{-1} \zeta_{\lambda}$ in $\hat{V}$ and the representation $\pi_{\lambda, \alpha, \delta}$ of $G_{\lambda}$. In the case that either $\pi_{\lambda, \alpha, \delta}$ or $\pi_{\alpha}$ are primary representations, the space of $F_{\lambda, \alpha, \delta}$ components will have multiplicity equal to the product of the multiplicities of $\pi_{\lambda, \alpha, \delta}$ and $\pi_{\alpha}$. 
5. Orthogonal Radon transformations. Fix values of $k$ and $k^{\prime}$ between 0 and $n-1$, and fix $j$ between $\max \left(0, k+k^{\prime}-n\right)$ and $\min \left(k, k^{\prime}\right)$. We are going to define a generalization of the Radon transform, $R\left(k^{\prime}, k, j\right)$, from functions on $P_{n, k^{\prime}}$ to functions on $P_{n, k}$, that intertwines the action of $G \times V$. Given a fixed $k$-plane $\pi$, consider all $k^{\prime}$-planes $\pi^{\prime}$ that intersect $\pi$ orthogonally in a $j$-plane. If the $k$-plane $\pi$ is $[\omega, y]$, where $\omega=g\left(\begin{array}{c}0 \\ I_{m}\end{array}\right)$, then it is not hard to see that all such $k^{\prime}$-planes are of the form

$$
\left[g\left(\begin{array}{ll}
s & 0 \\
0 & r
\end{array}\right),\left(\begin{array}{c}
z \\
r^{\mathrm{tr}} y
\end{array}\right)\right], \quad \text { where } s \in S_{k-j}^{k}, r \in S_{d}^{m}, \text { and } z \in \mathbf{R}^{k-j}
$$

for $d=m^{\prime}-k+j$ (note $0 \leq d \leq m$ ). Thus we define

$$
\begin{aligned}
R\left(k^{\prime}, k, j\right) f & \left(\left[g\left(\begin{array}{c}
0 \\
I_{m}
\end{array}\right), y\right]\right) \\
\quad= & \int_{\mathbf{R}^{k-j}} \int_{S_{d}^{m}} \int_{S_{k-j}^{k}} f\left(\left[g\left(\begin{array}{cc}
s & 0 \\
0 & r
\end{array}\right),\left(\begin{array}{c}
z \\
r^{\mathrm{tr}} y
\end{array}\right)\right]\right) d s d r d z
\end{aligned}
$$

for suitable functions $f$, and we will call $R\left(k^{\prime}, k, j\right)$ (or just $R$ ) an orthogonal Radon transform. It is easy to see that $R$ intertwines the actions of the Euclidean motion group. We expect that $R\left(k^{\prime}, k, j\right)$ is diagonalizable via harmonic analysis: If the harmonic analysis of $f$ is given by

$$
f=\int_{0}^{\infty} \sum_{\delta \in \Delta^{\prime}} F_{\lambda, \delta} d \nu(\lambda)
$$

then

$$
R f=\int_{0}^{\infty} \sum_{\delta \in \Delta^{\prime}} R F_{\lambda, \delta} d \nu(\lambda)
$$

is the harmonic analysis of $R f$. Of course the integrals in (5.2) and (5.3) must be taken in a suitably regularized sense, and the expression $R F_{\lambda, \delta}$ is formal, since the mild decay at $\infty$ of $F_{\lambda, \delta}$ will not always be enough to make the integral defining $R F_{\lambda, \delta}$ converge pointwise. However, if we can make sense of $R F_{\lambda, \delta}$, Schur's lemma assures us that the operator is uniquely determined up to a constant multiple.

Already, without doing any computation, (5.3) gives us a lot of information by comparing the set of highest weight vectors $\delta \in \Delta^{\prime}$ for $P_{n, k^{\prime}}$ with the set $\Delta$ for $P_{n, k}$. We have $\Delta^{\prime} \subseteq \Delta$ if and only if $\min \left(k^{\prime}, n-k^{\prime}-1\right) \leq \min (k, n-k-1)$, so this condition is necessary if we are to have $R$ invertible $\left(R F_{\lambda, \delta}=0\right.$ if $\delta \in \Delta^{\prime}$ but $\delta \notin \Delta)$. Similarly the reverse inequality is necessary if we are to have $R$ onto.

To get more information we need to compute $R F_{\lambda, \delta}$ for the function $F_{\lambda, \delta}$ given by (3.14) (or equivalently (3.15)), which we will denote $G_{\lambda, \delta}$ or $G_{\lambda, \delta}^{\prime}$ (for the choice of $k$ or $k^{\prime}$, respectively). We have

$$
R G_{\lambda, \delta}^{\prime}=c(\lambda, \delta) G_{\lambda, \delta}
$$

by Schur's lemma, and it is not hard to see that

$$
c(\lambda, \delta)=\lambda^{j-k} c(\delta)
$$


by considering the action of dilations (this will also come out of our computations). To compute $c(\delta)$ it suffices to evaluate $G_{\lambda, \delta}$ and $R G_{\lambda, \delta}^{\prime}$ at a single point for which $G_{\lambda, \delta}$ is nonzero.

Now by $(3.15)$ we have

$$
G_{\lambda, \delta}([\omega, 0])=\int_{O(m)} \tilde{M}_{\delta}(\omega s) d s
$$

and we choose $\omega=\tilde{\omega}$, where $\tilde{\omega}_{m}=e_{1}, \tilde{\omega}_{m-1}=e_{3}, \ldots$ Then $a_{p} \cdot \tilde{\omega}_{m-q+1}=\delta_{p}^{q}$, and so

$$
\tilde{M}_{\delta}(\tilde{\omega} s)=\prod_{i=1}^{\nu_{0}}\left(\operatorname{det}\left\{s_{p q}\right\}_{1 \leq p, q \leq i+1}\right)^{2 \lambda_{i}}
$$

(with a slight modification in the case $n$ odd, $m-1=k$ ) and we have the nonzero value

$$
G_{\lambda, \delta}([\tilde{\omega}, 0])=\int_{O(m)} \prod_{i=1}^{\nu_{0}}\left(\operatorname{det}\left\{s_{p q}\right\}_{1 \leq p, q \leq i+1}\right)^{2 \lambda_{i}} d s
$$

where $\lambda_{j}$ is given by (3.11) and depends only on $\delta$. Presumably there is an explicit formula evaluating this integral, but we have not been able to find it.

Next we compute $R G_{\lambda, \delta}^{\prime}([\tilde{\omega}, 0])$. Strictly speaking we should multiply by a test function $\psi(\lambda)$ supported away from the origin and integrate, for then $\int \psi(\lambda) G_{\lambda, \delta}^{\prime} d \lambda$ will be rapidly decreasing, so $R \int \psi(\lambda) G_{\lambda, \delta}^{\prime} d \lambda$ is well defined and

$$
R \int \psi(\lambda) G_{\lambda, \delta}^{\prime} d \lambda=\int c(\lambda, \delta) \psi(\lambda) G_{\lambda, \delta} d \lambda
$$

is the analogue of (5.4), from which we can recover $c(\lambda, \delta)$. However, it is simpler to compute formally but directly. Choose $\tilde{g} \in G$ such that $\tilde{g}\left(\begin{array}{c}0 \\ I_{m}\end{array}\right)=\tilde{\omega}$. Then

$$
\begin{aligned}
& R G_{\lambda, \delta}^{\prime}([\tilde{\omega}, 0]) \\
& \quad=\int_{O\left(m^{\prime}\right)} \int_{S_{d}^{m}} \int_{S_{k-j}^{k}} \int_{\mathbf{R}^{k-j}} \tilde{M}_{\delta}\left(\tilde{g}\left(\begin{array}{cc}
s & 0 \\
0 & r
\end{array}\right) t^{\prime}\right) e^{i \lambda\left\langle\left(\begin{array}{l}
z \\
0
\end{array}\right), t^{\prime} e_{m^{\prime}}\right\rangle} d z d s d r d t^{\prime} .
\end{aligned}
$$

Now we can perform the $z$ integration and obtain a factor $(2 \pi \lambda)^{j-k}$ times the $\delta$ function in the first $k-j$ variables of $t^{\prime} e_{m^{\prime}}$. However, since we can always change the $r$ variable by multiplying on the right by an element of $O(d)$, we can force the last column of $t^{\prime}$ to be

$$
\left(\begin{array}{c}
0 \\
\vdots \\
0 \\
1
\end{array}\right)
$$

and so

$$
\begin{aligned}
& R G_{\lambda, \delta}^{\prime}([\tilde{\omega}, 0]) \\
& \quad=(2 \pi \lambda)^{j-k} \int_{O\left(m^{\prime}-1\right)} \int_{S_{d}^{m}} \int_{S_{k-j}^{k}} \tilde{M}_{\delta}\left(\tilde{g}\left(\begin{array}{cc}
s & 0 \\
0 & r
\end{array}\right)\left(\begin{array}{cc}
t^{\prime \prime} & 0 \\
0 & 1
\end{array}\right)\right) d s d r d t^{\prime \prime}
\end{aligned}
$$


Further properties of $R$ will require a more explicit evaluation of the integrals in (5.6) in (5.7). In particular, we mention the question of whether or not (5.7) is zero. In the special case $j=0, n$ odd, and $k+k^{\prime}=n-1$, Gonzalez [3] has inverted $R$ by a different method.

We can similarly define orthogonal Radon transforms on the $n$-sphere $S^{n}=$ $S O(n+1) / S O(n)$. A $k$-plane in $S^{n}$ (a totally geodesic $k$-dimensional subspace) is the intersection of the sphere, embedded in $\mathbf{R}^{n-1}$ in the usual way, with a $k+1$-dimensional subspace through the origin in $\mathbf{R}^{n+1}$, so the space of $k$-planes is $G_{n+1, k+1}$, and an individual $k$-plane can be represented either by $g \in S O(n+1)$ or by $\omega=g\left(\begin{array}{c}0 \\ I_{m}\end{array}\right) \in S_{m}^{n+1}$, where $m+k=n$. As before we will write $[\omega]$ for the $k$-plane associated with $\omega$ (so as a point set in $S^{n}$ it is the set of vectors orthogonal to the $m$ columns of $\omega$ ).

Again we fix values $k$ and $k^{\prime}$ between 0 and $n-1$ and $j$ between $\max \left(0, k+k^{\prime}-n\right)$ and $\min \left(k, k^{\prime}\right)$, and consider for a fixed $k$-plane $\pi$ all the $k^{\prime}$-planes that intersect $\pi$ orthogonally in a $j$-plane. The integral transform from functions of $k^{\prime}$-planes to functions of $k$-planes associated with integrating over this set is given by

$$
R\left(k, k^{\prime}, j\right) f\left(\left[g\left(\begin{array}{c}
0 \\
I_{m}
\end{array}\right)\right]\right)=\int_{S_{d}^{m}} \int_{S_{k-j}^{k+1}} f\left(\left[g\left(\begin{array}{cc}
s & 0 \\
0 & r
\end{array}\right)\right]\right) d s d r
$$

where $d=m^{\prime}-k+j$. If $f=\sum_{\delta \in \Delta^{\prime}} F_{\delta}$ is the harmonic analysis of a function of $k^{\prime}$-planes (here $\Delta^{\prime}$ is the set of weights $\left(\delta_{1}, \ldots, \delta_{\nu_{0}^{\prime}}, 0, \ldots, 0\right)$ where

$$
\nu_{0}^{\prime}=\min \left(k^{\prime}+1, m^{\prime}\right)
$$

and $\delta_{i}$ are even), then $R f=\sum_{\delta \in \Delta^{\prime}} R F_{\delta}$ is the harmonic analysis of $R f$. Furthermore, if for $F_{\delta}$ we choose the marked function (see $\S 6$ )

$$
G_{\delta}^{\prime}([\omega])=\int_{O\left(m^{\prime}\right)} M_{\delta}^{\prime}(\omega t) d t
$$

where

$$
M_{\delta}^{\prime}(\omega)=\prod_{i=1}^{\nu_{0}^{\prime}} \operatorname{det}\left\{a_{p} \cdot \omega_{m^{\prime}-q+1}\right\}_{1 \leq p, q \leq i}^{2 \lambda_{i}}
$$

with $2 \lambda_{i}=\delta_{i}-\delta_{i+1}$ (with the obvious modification in the case $k^{\prime}+1=m^{\prime}$ and $\left.\delta_{\nu_{0}^{\prime}}<0\right)$, then $R G_{\delta}^{\prime}=c(\delta) G_{\delta}$. If we choose $\omega=\tilde{\omega}$ as before then in analogy with (5.6) we have

$$
G_{\delta}([\tilde{\omega}])=\int_{O(m)} \prod_{i=1}^{\nu_{0}}\left(\operatorname{det}\left\{s_{p q}\right\}_{1 \leq p, q \leq i}\right)^{2 \lambda_{i}} d s
$$

and also

$$
R G_{\delta}^{\prime}([\tilde{\omega}])=\int_{O\left(m^{\prime}\right)} \int_{S_{d}^{m}} \int_{S_{k-j}^{k+1}} M_{\delta}\left(\left[\tilde{g}\left(\begin{array}{cc}
s & 0 \\
0 & r
\end{array}\right) t^{\prime}\right]\right) d s d r d t^{\prime} .
$$

6. Concluding remarks. (1) There is some confusion in our previous paper [10] concerning the representations that occur in $L^{2}$ of the Grassmannian manifolds, arising from our failure to distinguish between the Grassmannian $G_{n, k}$ (either as $S O(n) / S(O(m) \times O(k))$ or $O(n) / O(m) \times O(k))$ and the larger space $S O(n) / S O(m) \times S O(k)$. Also, we now have a very elegant result due to Helgason 
$[6,7]$, giving the integrality condition on the highest weight $\delta=\left(\delta_{1}, \delta_{2}, \ldots, \delta_{\nu}\right)$ which is necessary and in some cases sufficient for the representation $\pi_{\delta}$ to appear in $G / K$ for any compact symmetric space, where $G$ and $K$ are both connected, as follows. Let $G_{\mathbf{C}}$ denote the complexification of $G$, and let $\tilde{G}$ be a connected real-form such that $\tilde{G} / K$ is the dual symmetric space to $G / K$. Choose a maximal torus $T$ in $G$ which splits $T=T_{1} \oplus T_{2}$ with $T_{2} \subseteq K$ and such that the complexification of $T_{1}$ intersects $\tilde{G}$ in the abelian component $\tilde{A}$ of the Iwasawa decomposition of $\tilde{G}$. Then Helgason's condition is that $\delta$ restricted to $T_{2}$ must be zero, i.e., $\delta=\left(\delta_{1}, \ldots, \delta_{\nu_{0}}, 0, \ldots, 0\right)$, where $\nu_{0}$ is the dimension of $T_{1}$ and in addition

$$
\frac{\langle\delta, \alpha\rangle}{\langle\alpha, \alpha\rangle} \in \mathbf{Z}^{+} \quad \text { for all } \alpha \in \Delta^{+}
$$

where $\Delta^{+}$is the set of positive roots for $\tilde{G}$. In [7] Helgason proves this is necessary and sufficient under the assumption that $G$ is simply-connected. However, the necessity is easily seen as follows (this argument is given in Flested-Jensen [2, p. 180], but he also asserts that it is sufficient without giving a convincing proof). If $\pi_{\delta}$ appears in $G / K$, then the holomorphic extension of $\pi_{\delta}$ to $G_{\mathbf{C}}$ when restricted to $\tilde{G}$ yields a finite-dimensional representation $\tilde{\pi}_{\delta}$ of $\tilde{G}$ with a $K$-fixed vector, and $\tilde{\pi}_{\delta}$ determines $\pi_{\delta}$ uniquely. But then the results of Helgason [6] describe all such representations as being given by highest weights (on $\tilde{A}$ ) satisfying $(6.1$ ), and it is easy to see that the weight must be the restriction of $\delta$ to $\tilde{A}$. We do not need the sufficiency of $(6.1)$ because we can explicitly construct the representations.

In our case $G=S O(n), K=S O(m) \times S O(k)$ with say $\nu_{0}=m \leq k$. The group $\tilde{G}$ is $S O_{e}(m, k)$, and $\tilde{A}=\exp a(t)$ for $t \in T^{m}$, where

$$
a(t)=\left(\begin{array}{ccc}
0 & \operatorname{diag}(t) & 0 \\
\operatorname{diag}(t) & 0 & 0 \\
0 & 0 & 0
\end{array}\right)
$$

and $\operatorname{diag}(t)$ denotes the $m \times m$ diagonal matrix with diagonal entries $t_{1}, \ldots, t_{m}$, and the roots are easily computed to be $t=\left(t_{1}, \ldots, t_{m}\right) \rightarrow \pm t_{r} \pm t_{s}$ for $r \neq s$ with multiplicity one, and $t \rightarrow \pm t_{r}$ with multiplicity $k-m$. The usual lexicographic ordering yields the positive roots $t \rightarrow t_{r} \pm t_{s}$ for $r<s$ and $t \rightarrow t_{r}$ (if $k>m$ ). Thus (6.1) says $\delta_{r} \pm \delta_{s} \in 2 \mathbf{Z}^{+}$and $\delta_{s} \in \mathbf{Z}^{+}$if $k>m$. In addition to the usual conditions for a dominant weight for $S O(n), \delta_{j}$ integers and $\delta_{1} \geq \delta_{2} \geq \cdots \geq \delta_{m} \geq 0$ if $k>m\left(\delta_{1} \geq \delta_{2} \geq \cdots \geq \delta_{m-1} \geq\left|\delta_{m}\right|\right.$ if $\left.k=m\right)$, we have the additional requirement that all the $\delta_{j}$ for $j=1, \ldots, m$ have the same parity (this condition is misstated in $[\mathbf{1 0}])$.

Now if we wish to realize the highest weight vector for each such representation as a function on $S_{m}^{n}$ invariant under the action on the right by $S O(m)$ we take

$$
G_{\delta}(\omega)=\int_{S O(m)} M_{\delta}(\omega t) d t
$$

where

$$
M_{S}(\omega)=\prod_{i=1}^{\nu_{0}} \operatorname{det}\left\{a_{p} \cdot \omega_{q}\right\}_{1 \leq p, q \leq i}^{\delta_{i}-\delta_{i+1}}
$$


(in the case $k=m$ and $\delta_{m}<0$ we replace $a_{m}$ by $\overline{a_{m}}$ and take $\left|\delta_{m}\right|$ as the exponent when $i=m)$. The fact that $M_{\delta}(\omega)$ is a highest weight vector for $\delta$ on $S_{m}^{n}$ is established in [10], and the invariance is clear from (6.2). The fact that we have a nonzero value for $G_{\delta}(\omega)$ for $\omega=\tilde{\omega}$ as in $\S 5$ uses the fact that $\delta_{i}-\delta_{i+1}$ is even for $i<\nu_{0}=m$, and $\operatorname{det}\left\{a_{p} \cdot \omega_{q}\right\}_{1 \leq p, q \leq m}$ is invariant under $\omega \rightarrow \omega t$ for $t \in S O(m)$. In $[10]$ we used a summation rather than the integral in (6.2).

Now to get functions on the Grassmannian we need to have invariance under $O(m)$, and clearly this depends oon the parity of $\delta_{m}$ being even. Altogether then we require

$$
\delta=\left(\delta_{1}, \ldots, \delta_{\nu_{0}}, 0, \ldots, 0\right) \text { with } \delta_{1}, \ldots, \delta_{\nu_{0}} \text { even, }
$$

and then (6.2) and (6.3) give an explicit realization of a nonzero highest weight vector. Notice that we no longer require $m \leq k$ here (we cannot dispense with this hypothesis when $\delta_{\nu_{0}}$ is odd, however, for then the integral in (6.2) will vanish identically). We could also take the integral in $(6.2)$ over $O(m)$ rather than $S O(m)$.

Helgason's theorem also provides a formula for the zonal spherical function of the representation $\pi_{\delta}$ in $G / K$. This might be quite useful for some applications, but it does not provide a "marked" vector that can be compared when we vary $K$.

(2) We have dealt with Grassmannian manifolds and bundles over the real number field $\mathbf{R}$, but everything generalizes to the complex and quaternion fields. Here we briefly indicate the modifications in the complex case. The Grassmannian manifold $G \mathbf{C}_{n, k}$ of complex $k$-dimensional subspaces of $\mathbf{C}^{n}$ can be regarded as $G / H$ with $G=S U(n)$ and $H=S(U(k) \times U(m))$, where $k+m=n$, or we could take $G=U(u)$ and $H=U(k) \times U(m)$. We will take the latter case even though $U(n)$ is not semisimple since it contains more information (every irreducible representation of $U(n)$ is already irreducible in $S U(n)$, but restriction to $S U(n)$ does not determine the representation on $U(n))$, and it is somewhat simpler.

The Grassmannian bundle then is constructed by taking $V=\mathbf{C}^{n}, \rho$ as the standard representation of $G$ on $V$, and $W=\mathbf{C}^{k}$. The orbits of $\rho(H)$ on $W^{\perp}$ are again spheres parametrized by the positive radius $\lambda>0$, and if

$$
z_{\lambda}=\left(\begin{array}{c}
0 \\
\vdots \\
0 \\
\lambda
\end{array}\right)
$$

then

$$
G_{\lambda}=U(n-1) \text { and } H_{\lambda}=U(k) \times U(m-1) .
$$

We choose the diagonal matrices as a maximal torus in $G$ and $G_{\lambda}$; these have dimensions $n$ and $n-1$, respectively. Irreducible representations of $G_{\lambda}$ are indexed by highest weight vectors $\delta=\left(\delta_{1}, \ldots, \delta_{n-1}\right)$, where the $\delta_{j}$ are integers satisfying $\delta_{1} \geq \delta_{2} \geq \cdots \geq \delta_{n-1}$. Those which are of class one with respect to $H_{\lambda}$ are of the form $\left(\delta_{1}, \ldots, \delta_{\nu_{0}}, 0, \ldots, 0,-\delta_{\nu_{0}}, \ldots,-\delta_{1}\right)$, where $\nu_{0}=\min (k, m-1)$ and there are $n-1-2 \nu_{0}$ zeros, and the highest weight vector, realized as a function on the Stiefel manifold $U_{m-1}^{n-1}=U(n-1) / U(k)$ which are invariant under the right action 
of $U(m-1)$, are given by $\int_{U(m-1)} M_{\delta}\left(\omega_{s}\right) d s$ where

$$
M_{\delta}(\omega)=\prod_{i=1}^{\nu_{0}}\left(\operatorname{det}\left\{\omega_{p, q}\right\}_{q \leq p, q \leq i} \operatorname{det}\left\{\bar{\omega}_{n-p, q}\right\}_{1 \leq p, q \leq i}\right)^{\delta_{i}-\delta_{i+1}}
$$

(see $[11$, p. 321]).

Now we introduce coordinates $[\omega, z]$ on the Grassmannian bundle with $\omega \in U_{m}^{n}$ and $z \in \mathbf{C}^{m}$ for the $k$-plane $\omega^{\perp}+\omega z$. The analogue of (3.15) is now

$$
F_{\lambda, \delta}([\omega, z])=\int_{U(m)} \tilde{M}_{\delta}(\omega s) e^{i \lambda \operatorname{Re}\left\langle z, s e_{m}\right\rangle} d s
$$

where

$$
\tilde{M}_{\delta}(\omega)=\prod_{i=1}^{\nu_{0}}\left(\operatorname{det}\left\{\omega_{m+1-p, q}\right\}_{1 \leq p, q \leq i+1} \operatorname{det}\left\{\bar{\omega}_{m+1-p, n+1-q}\right\}_{1 \leq p, q \leq i+1}\right)^{\delta_{i}-\delta_{i+1}} .
$$

The function $F_{\lambda, \delta}$ is marked as the highest weight vector for the representation of $G$ with highest weight

$$
\left(\delta_{1}, \delta_{1}, \delta_{2}, \ldots, \delta_{\nu_{0}}, 0, \ldots, 0,-\delta_{\nu_{0}}, \ldots,-\delta_{2},-\delta_{1},-\delta_{1}\right)
$$

with $n-2 \nu-2$ zeros unless $k=m-1=\nu_{0}$, in which case the weight is

$$
\left(\delta_{1}, \delta_{1}, \delta_{2}, \ldots \delta_{\nu_{0}-1}, 0,-\delta_{\nu_{0}-1}, \ldots,-\delta_{2},-\delta_{1},-\delta_{1}\right)
$$

(in the case $n=3$ it is just $\left.\left(\delta_{1}, 0,-\delta_{1}\right)\right)$. There are analogous orthogonal Radon transforms as well. We leave the details to the interested reader. For compact analogues see Grinberg [5].

(3) The invariant differential operators on the Grassmannian bundle have recently been determined by Gonzalez and Helgason [4]. They find $\nu_{0}+1$ generators, which is consistent with the fact that there are $\nu_{0}+1$ parameters among $\lambda$ and $\delta$, and suggests that this is the natural "rank" of the space. It would be interesting to diagonalize these operators.

(4) There is no invariant Riemannian metric on the Grassmannian bundle, nor is there an invariant semi-Riemannian metric. This fact has significant consequences for the harmonic analysis; in particular, it means that we cannot control the smoothness of a function merely by the decay of its Fourier components. Thus while we should expect a simple Paley-Weiner characterization of the Fourier components of functions in $D$ or $S$, the characterization will involve smoothness of the components as well as decay in $\lambda$ and $\delta$.

Another consequence is that there is no natural definition of Sobolev spaces. Of course we can define quasi-invariant Riemannian metrics (so that the group actions are Lipschitz continuous) and Sobolev spaces based on these, but it is not even clear whether there is a natural equivalence class of quasi-invariant metrics. Of course one can define Sobolev spaces where only derivatives in the fiber directions are allowed, and these spaces might enter into a Plancherel formula for orthogonal Radon transforms.

(5) The Grassmannian bundle $P_{n, k}$ can be thought of as a limit of Grassmannian manifolds $G_{n+1, k+1}$ of $k$-planes in $S^{n}$ as the radius of the sphere tends to infinity. In the case of points, $k=0$, the harmonic analysis on the sphere $S^{n}$ and the harmonic analysis on $\mathbf{R}^{n}$ are related by the well-known Mehler-Heine identities. 
One would similarly expect there to be limiting relations connecting the harmonic analysis of $G_{n+1, K+1}$ and $P_{n, k}$.

One can also obtain $P_{n, k}$ as a limit from the "other side." Namely, consider the hyperbolic Grassmannian bundle of totally geodesic $k$-dimensional submanifolds of an $n$-dimensional hyperbolic space of constant curvature. This can be identified with $O(n, 1) / O(m) \times O(k, 1)$ which is a semisimple symmetric space, and $P_{n, k}$ is obtained in the limit as the curvature tends toward zero. Very little is known about the harmonic analysis of this semisimple symmetric space, and one can hope that some light will be shed on this problem by the results of this paper.

\section{REFERENCES}

1. M. Berger, Les espaces symétriques non compact, Ann. Sci. École Norm. Sup. (3) 74 (1957), 85-177.

2. M. Flensted-Jensen, Harmonic analysis on semisimple symmetric spaces, Proc. 1982-83 Maryland Conf., Vol. III (R. Herb et al., eds.), Lecture Notes in Math., vol. 1077, SpringerVerlag, Berlin and New York, 1984, pp. 166-209.

3. F. Gonzalez, Radon transforms on Grassmann manifolds, thesis, MIT, 1984.

4. F. Gonzalez and S. Helgason, Invariant differential operators on Grassmann manifolds, preprint.

5. E. Grinberg, Spherical harmonics and integral geometry on projective spaces, Trans. Amer. Math. Soc. 279 (1983), 187-203.

6. S. Helgason, $A$ duality for symmetric spaces with applications to group representations. I, Adv. Math. 5 (1970), 1-154.

7. __ Some results on eigenfunctions on symmetric spaces and eigenspace representations, Math. Scand. 41 (1977), 79-89.

8. G. W. Mackey, The theory of unitary group representations, Univ. Chicago Press, 1976.

9. T. Oshima, Fourier analysis on semisimple symmetric spaces, Proc. Marseille-Luminy 1980 Conf. (J. Carmona and M. Vergne, eds.), Lecture Notes in Math., vol. 880, SpringerVerlag, Berlin and New York, 1981, pp. 357-369.

10. R. Strichartz, The explicit Fourier decomposition of $L^{2}(S O(n) / S O(n-m))$, Canad. J. Math. 24 (1972), 915-925.

11. __ Bochner identities for Fourier transforms, Trans. Amer. Math. Soc. 228 (1977), 307-327.

12. $\ldots, L^{p}$ estimates for Radon transforms in Euclidean and non-Euclidean spaces, Duke Math. J. 48 (1981), 699-727.

DePaRtMent of Mathematics, CORNELl University, IthaCA, NEW YoRK 14853 